\title{
Gauge Theory for a Doped Antiferromagnet in a Rotating Reference-Frame
}

\author{
C. Kübert and A. Muramatsu \\ Institut für Physik, Universität Augsburg \\ Memmingerstr. 6, 86135 Augsburg, Germany
}

(May 5, 1995)

\begin{abstract}
We study a doped antiferromagnet $(\mathrm{AF})$ using a rotating reference-frame. Whereas in the laboratory reference-frame with a globally fixed spin-quantization axis (SQA) the long-wavelength, lowenergy physics is given by the $\mathrm{O}(3)$ non-linear $\sigma$-model with current-current interactions between the fermionic degrees of freedom and the order-parameter field for the spin-background, an alternative description in form of an $\mathrm{U}(1)$ gauge theory can be derived by choosing the SQA defined by the local direction of the order-parameter field via a $\mathrm{SU}(2)$ rotation of the fermionic spinor. Within a large- $N$ expansion of this $\mathrm{U}(1)$ gauge theory we obtain the phase diagram for the doped AF and identify the relevant terms due to doping that lead to a quantum phase transition at $T=0$ from the antiferromagnetically ordered Néel phase to the quantum-disordered (QD) spin-liquid phase. Furthermore, we calculate the propagator of the corresponding U(1) gauge field, which mediates a long-range transverse interaction between the bosonic and fermionic fields. It is found that the strength of the propagator is proportional to the gap of the spin-excitations. Therefore, we expect as a consequence of this long-range interaction the formation of bound states when the spin-gap opens, i.e. in the QD spin-liquid phase. The possible bound states are spin-waves with a (spin-) gap in the excitation spectrum, spinless fermions and pairs of fermions. Thus, an alternative picture for charge-spin separation emerges, with composite charge-separated excitations. Moreover, the present treatment shows an intimate connection between the opening of the spin-gap and charge-spin separation as well as pairing.
\end{abstract}

PACS: 71.27.+a, 74.20.Mn, 74.25.Ha

\section{INTRODUCTION}

Recently much attention has been focused on the problem of understanding the magnetic as well as transport properties of high-temperature superconductors (HTSC) as a function of doping. The undoped parent compounds like $\mathrm{La}_{2} \mathrm{CuO}_{4}$ are antiferromagnetic insulators with $S=1 / 2$ local moments on the copper-sites [1]. Upon doping chargecarriers into the $\mathrm{CuO}_{2}$ planes, the long-range magnetic order is destroyed and these materials become superconducting at low temperatures, while in the normal state unconventional properties are observed [2]. The particular interesting question arising here is whether these anomalous features are directly related to the proximity to the magnetic quantum phase-transition or not.

Since in the relevant low-doping regime the spin-spin correlation length $\xi$ is large [3], it is appropriate to address this question from a field-theoretic point of view. Therefore we will study this problem within an effective long-wavelength, low-frequency theory, which can be derived in a rigorous way from a realistic microscopic model, i. e. without semiclassical approximations and phenomenological assumptions. By means of a SU(2) rotation of the fermionic spinor one can further derive two alternative descriptions of the doped AF [4]. In the laboratory reference-frame with a globally fixed SQA, the doped AF is described by the $\mathrm{O}(3)$ non-linear $\sigma$-model for the order-parameter field $\boldsymbol{n}$ of the spin-background and current-current interactions between the doped fermions and the order-parameter field. On the other hand, in the rotating reference-frame with local SQA defined by the local direction of $\boldsymbol{n}$, the long-wavelength low-energy theory for the doped AF is given by an $\mathrm{U}(1)$ gauge theory with bosonic and fermionic degrees of freedom minimally coupled to an $\mathrm{U}(1)$ vector gauge field. The present work presents an analysis of the gauge theory in the rotating reference-frame.

Within a large- $N$ expansion of the $\mathrm{U}(1)$ gauge theory we will explicitly show 1 ) that there occurs a quantum phasetransition from the antiferromagnetically ordered Néel-state to a QD spin-liquid phase at $T=0$ induced by doping and 2) that the U(1) gauge field mediates a long-range interaction between the fermionic and bosonic fields, where the strength of the coupling is given by the spin-gap. Therefore, bound states between excitations with opposite charge (as defined by the coupling to the gauge field) are expected to form in the QD phase. The possible bound states correspond to spin-waves with a gap in the excitation spectrum, spinless fermions and pairs of fermions. In this frame the phenomena of charge-spin separation and pairing are intimately connected with the opening of a spin-gap and have the same physical origin, i. e. the long-range interaction induced by the vector gauge field.

In order to clarify the connection of the gauge theory studied here with a microscopic model, we summarize in the 
following the main steps involved to reach the effective field-theory [4]. Our starting point is the strong coupling limit of the three-band Hubbard model for the $\mathrm{CuO}_{2}$ planes, which is given by the spin-fermion model [5] and acts in the subspace of singly occupied $\mathrm{Cu}$-sites:

$$
\hat{H}=\sum_{\substack{<j, k>\\ \sigma}} t_{j k} c_{j, \sigma}^{\dagger} c_{k, \sigma}+J_{K} \sum_{i} \boldsymbol{R}_{i} \cdot \boldsymbol{S}_{i}+J_{H} \sum_{<i, i^{\prime}>} \boldsymbol{S}_{i} \cdot \boldsymbol{S}_{i^{\prime}}
$$

where $c_{j, \sigma}^{\dagger}$ and $c_{j, \sigma}$ create and annihilate holes with spin-projection $\sigma=\uparrow, \downarrow$ in an oxygen-orbital. The kinetic term describes hopping processes between oxygen-sites and can include a direct oxygen-oxygen hopping as well as an effective hopping via the central copper-site. Furthermore the Hamiltonian contains a non-local, Kondo-like interaction between the localized Cu-spins $\boldsymbol{S}_{i}$ and the neighboring O-holes $\boldsymbol{R}_{i}=\sum_{\substack{(j, k ; i) \\ \alpha, \beta}}(-1)^{\alpha_{i j}+\alpha_{i k}} c_{j, \alpha}^{\dagger} \boldsymbol{\sigma}_{\alpha \beta} c_{k, \beta}$ (here the summation goes only over the indices $j$ and $k$ denoting the oxygen-sites around the central copper-site $i$ ), where $\sigma$ are the Pauli matrices, as well as an antiferromagnetic Heisenberg superexchange interaction between nearest neighbor $\mathrm{Cu}$-spins. For a connection of the parameters of the spin-fermion model with the ones of the three-band Hubbard model as well as the definitions of the phase factors $\alpha_{i j}$ which accounts for the d- and p-wave symmetry of the underlying orbitals we refer to Ref. 沺.

In order to construct an effective theory for the long-wavelength low-energy sector of the spin-fermion model we have to integrate out all the high-energy modes of the model. For the spin part this is done by means of an expansion around the adiabatic limit within the method of generalized Berry phases [1,66. The continuum-limit in the orderparameter field $\phi_{i}$ of the spin-background is achieved by means of a gradient-expansion around the AF saddle-point $\phi_{i}=(-1)^{i} \boldsymbol{n}_{i}$, where the lattice constant $a$ (or the inverse of an equivalent ultraviolet cutoff) serves as the small expansion parameter. Consequently, the Kondo-like interaction in the Hamiltonian Eq. (11) goes over to essentially the same form but with the order-parameter $\boldsymbol{n}_{i}$ instead of the spin-operator $\boldsymbol{S}_{i}$. Therefore it becomes a staggered potential for the fermions in the antiferromagnetic ground-state and together with the hopping parts it determines the dispersion of the fermions which has minima located at $\boldsymbol{k}_{\min }=( \pm \pi / 2, \pm \pi / 2)$. Since we are interested in the low doping regime and low-energy sector of the model it is justified to substitute the whole band structure by parabolic bands located at $\boldsymbol{k}_{\min }$. In this way we obtain the following continuum theory:

$$
\begin{aligned}
S_{N l \sigma} & =\frac{1}{2 g} \int_{0}^{\beta} d \tau \int d^{2} r\left[\left(\partial_{r} \boldsymbol{n}(\boldsymbol{r}, \tau)\right)^{2}+\frac{1}{c^{2}}\left(\partial_{\tau} \boldsymbol{n}(\boldsymbol{r}, \tau)\right)^{2}\right], \\
S_{F} & =\int_{0}^{\beta} d \tau \int d^{2} r\left[c^{\dagger} \partial_{\tau} c+\frac{1}{2 m_{r}} \partial_{r} c^{\dagger} \partial_{r} c-\gamma_{r}\left(\partial_{r} \boldsymbol{n}\right)^{2} c^{\dagger} c+\sum_{\mu} \gamma_{\mu} \boldsymbol{J}_{\mu}^{S} \cdot \boldsymbol{J}_{\mu}^{F}\right] ; \mu=(r, \tau)
\end{aligned}
$$

where the first two terms in $S_{F}$ describe the kinetics of the fermions with perpendicular $m_{\perp}$ and parallel $m_{\|}$masses with respect to the borders of the magnetic Brillouin-zone. The remaining parts denote the interaction of the fermions with the antiferromagnetic spin-background, whose dynamics is controlled by the $\mathrm{O}(3)$ non-linear $\sigma$-model with $g=2 \sqrt{2} a / s$ being the coupling constant and $c=2 \sqrt{2} a J_{H} s$ being the spin-wave velocity. $s$ denotes here the length of the spin. The third term of $S_{F}$ gives a renormalization of the spin-stiffness of the non-linear $\sigma$-model, whenever the corresponding site is occupied by a hole. The fourth term gives current-current couplings similar to the ones obtained by Shraiman and Siggia for the $t-J$ model [7]. For the temporal component we have a coupling of the local spindensity $\boldsymbol{J}_{\tau}^{F}=c^{\dagger} \boldsymbol{\sigma} c$ of the fermions to the background magnetization $\boldsymbol{J}_{\tau}^{S}=i \boldsymbol{n} \times \partial_{\tau} \boldsymbol{n}$ whereas the spatial components denotes a coupling between the spin-current of the holes $\boldsymbol{J}_{r}^{F}=i\left(\partial_{r} c^{\dagger} \boldsymbol{\sigma} c-c^{\dagger} \boldsymbol{\sigma} \partial_{r} c\right)$ and the magnetization current of the background $\boldsymbol{J}_{r}^{S}=\boldsymbol{n} \times \partial_{r} \boldsymbol{n}$. The connections of the corresponding coupling constants as well as the masses to the microscopic parameters of the spin-fermion model are given in Ref. [4]. For simplicity we will use isotropic parameters $\gamma=\gamma_{\perp}=\gamma_{\|}$and $m=m_{\perp}=m_{\|}$in the following. However, such a situation is close to the case given by a parameter set for the underlying three-band Hubbard model, which, as quantum Monte Carlo simulations showed [8], is appropriate for a quantitative description of various physical quantities of the HTSC.

Alternatively one can describe the system using a reference-frame, which is defined by a local SQA for the fermionic spinor in the direction of the order-parameter field $\boldsymbol{n}$. This change of reference-frame is defined by the following local $S U(2)$ rotation of the fermionic spinor

$$
p(\boldsymbol{r}, \tau)=U^{\dagger}(\boldsymbol{r}, \tau) c(\boldsymbol{r}, \tau)
$$

where the $S U(2)$ rotation $U$ must fulfill $U^{\dagger}(\boldsymbol{r}, \tau) \boldsymbol{\sigma} \cdot \boldsymbol{n}(\boldsymbol{r}, \tau) U(\boldsymbol{r}, \tau)=\sigma^{z}$. Using a $C P^{1}$ representation [9] for the rotation matrix $U$ 


$$
U=\left(\begin{array}{cc}
z_{1} & -\bar{z}_{2} \\
z_{2} & \bar{z}_{1}
\end{array}\right)
$$

with $\bar{Z} Z=1$ and $\bar{Z}=\left(\bar{z}_{1}, \bar{z}_{2}\right)$ denoting a bosonic spinor, the transition to the rotating reference-frame is achieved by the following set of equations:

$$
\begin{aligned}
\text { uniform SQA } & \longleftrightarrow \text { rotating SQA } \\
c_{\mu} & \longleftrightarrow p_{\mu} \\
\partial_{\mu}-\frac{i}{2} \boldsymbol{\sigma} \cdot\left(\boldsymbol{n} \times \partial_{\mu} \boldsymbol{n}\right) & \longleftrightarrow \partial_{\mu}+i A_{\mu} \sigma^{z} \\
\frac{i}{2} \boldsymbol{\sigma} \cdot\left(\boldsymbol{n} \times \partial_{\mu} \boldsymbol{n}\right) & \longleftrightarrow K_{\mu}=i B_{\mu} \sigma^{-}+i \bar{B}_{\mu} \sigma^{+}
\end{aligned}
$$

Here we have introduced a composite gauge field $A_{\mu}$ and denoted the off-diagonal contributions by $B_{\mu}$ and $\bar{B}_{\mu}$ :

$$
\begin{aligned}
& A_{\mu}=-i \bar{Z} \partial_{\mu} Z \\
& B_{\mu}=i\left(z_{2} \partial_{\mu} z_{1}-z_{1} \partial_{\mu} z_{2}\right)=Z^{T} \sigma^{y} \partial_{\mu} Z, \\
& \bar{B}_{\mu}=i\left(\bar{z}_{1} \partial_{\mu} \bar{z}_{2}-\bar{z}_{2} \partial_{\mu} \bar{z}_{1}\right)=-\bar{Z} \sigma^{y} \partial_{\mu} \bar{Z}^{T},
\end{aligned}
$$

which are related to the $S U(2)$ rotation via $U^{\dagger} \partial_{\mu} U=i \sigma^{z} A_{\mu}+i \bar{B}_{\mu} \sigma^{+}+i B_{\mu} \sigma^{-}$. As a result of this transformation we get the following fermionic action

$$
S_{F}=\int_{0}^{\beta} d \tau \int d^{2} r\left\{p^{\dagger}\left(D_{\tau}^{F}+2 \tilde{\gamma}_{\tau} K_{\tau}\right) p+\frac{1}{2 m} \bar{D}_{r}^{F} p^{\dagger} D_{r}^{F} p+\tilde{\gamma}\left[2\left(\partial_{r} p^{\dagger} K_{r} p-p^{\dagger} K_{r} \partial_{r} p\right)-4 p^{\dagger} K_{r} K_{r} p\right]\right\}
$$

with $D_{\mu}^{F}=\partial_{\mu}+i \sigma^{z} A_{\mu},(\mu \in(\tau, \boldsymbol{r}))$ denoting a covariant derivative for the fermions and $\tilde{\gamma}_{\tau}=\gamma_{\tau}+1$ and $\tilde{\gamma}=\gamma+1 / 2 m$. Accordingly the $\mathrm{O}(3)$ non-linear $\sigma$-model transforms within this scheme by applying the Hopf projection 10 defined by

$$
\boldsymbol{n}=\bar{Z} \boldsymbol{\sigma} Z, \boldsymbol{n}^{2}=1 \Leftrightarrow \bar{Z} Z=1
$$

to Eq. (2) into the $C P^{1}$ model

$$
S_{C P^{1}}=\frac{2}{g} \int_{0}^{\beta} d \tau \int d^{2} r\left\{\bar{D}_{r}^{B} \bar{Z} D_{r}^{B} Z+\frac{1}{c^{2}} \bar{D}_{\tau}^{B} \bar{Z} D_{\tau}^{B} Z\right\}
$$

where $D_{\mu}^{B}=\partial_{\mu}-i A_{\mu}$ denotes a covariant derivative for the bosons. Here and in the rest of the paper the superscripts $F$ and $B$ refer to the contributions of the bosons and the fermions, respectively. The whole action in the rotating reference-frame $S=S_{F}+S_{C P^{1}}$ is invariant under a $U(1)$ gauge transformation.

From the definitions of covariant derivatives we get the following charges of the bosons and fermions with respect to the gauge field $A_{\mu}$ :

$$
\begin{array}{|c|c|}
\hline & \text { charge } \\
\hline z_{1} & +1 \\
z_{2} & +1 \\
p_{\uparrow} & +1 \\
p_{\downarrow} & -1 \\
\hline
\end{array}
$$

The gauge theory defined by Eqs. (10) and (12) is the starting point for the considerations in the present work.

The outline of the paper is as follows. Section $I$ is devoted to a large- $N$ expansion of the U(1) gauge theory, where we will show 1) that the theory describes a doping induced quantum phase transition from an antiferromagnetically ordered state to a quantum disordered spin-liquid state (Sec.II A) and 2) that the transverse gauge fields that mediate a long-range interaction between the bosons and fermions has a strength given by the mass of the $Z$-bosons, i.e. the spin-gap (Sec.IIB). In Section III we will discuss the physical consequences of the gauge field and consider the connection between the opening of a spin-gap and charge-spin separation and pairing. In this section we will also address the implications of the bound states on various physical properties of the cuprates in the low-doping regime and we will give a brief summary of our main results and conclusions. A short account of the results above is given in Ref. [11]. 


\section{LARGE- $N$ EXPANSION FOR THE GAUGE THEORY}

The gauge field $A_{\mu}$ is not an independent dynamical degree of freedom since it is composed by two $Z$-bosons and has no kinetic part in the action (10). Nevertheless, as usual [12], such a kinetic term will arise due to the fluctuations of the bosonic as well as of the fermionic components. The calculation of such fluctuations is, however, not straightforward since the coupling constant for the gauge fields is of order unity, implying that there exists no small expansion parameter in the theory. In order to overcome these difficulties a large- $N$ expansion is implemented, where $N$ is the number of bosonic or fermionic flavors [12].

Within the large- $N$ expansion it will be shown that doping drives the system from an antiferromagnetically ordered state at $T=0$ to a quantum disordered spin-liquid state. A mass for the $Z$-bosons is generated dynamically, such that a "spin-gap" enters naturally in the description of the low-energy properties of the system, much in the same way as in recent analysis of the $O(3)$ non-linear $\sigma$-model [13,14]. In the present case, however, the non-diagonal contributions $\propto K_{\mu}$ will lead to a doping dependence of the mass and, hence, a doping induced quantum phase-transition takes place. In the course of the large- $N$ expansion also the dynamics of the gauge fields is obtained, where the new energyscale generated dynamically (i.e. the mass for the $Z$-bosons) determines the strength of the propagator of the gauge fields. This fact reveals an intimate connection between the spin-gap and the properties of the charge-carriers in the QD phase.

As a first step we introduce $L$ copies of fermions with spin $\uparrow$ and with spin $\downarrow$

$$
\left(p_{\uparrow}, p_{\downarrow}\right) \longrightarrow\left(p_{\uparrow}^{1}, \ldots, p_{\uparrow}^{L} ; p_{\downarrow}^{1}, \ldots, p_{\downarrow}^{L}\right) .
$$

Accordingly the $C P^{1}$ fields are generalized to $C P^{N-1}$ ones

$$
\bar{Z}=\left(\bar{z}_{1}, \bar{z}_{2}\right) \longrightarrow \bar{Z}=\left(\bar{z}_{1}, \ldots, \bar{z}_{N}\right)
$$

with $N=2 L$ so that the ratio of fermionic and bosonic degrees of freedom remains the same after this generalization.

We proceed further by introducing sources for the fields and obtain (after proper renormalization of the fields) the following generating functional

$$
\begin{aligned}
& \mathcal{Z}\left[J, \bar{J}, \eta, \bar{\eta}, Q_{\mu}\right]=\int \mathcal{D} Z \mathcal{D} \bar{Z} \mathcal{D} p \mathcal{D} p^{\dagger} \prod_{\boldsymbol{r}, \tau} \delta\left(\bar{Z} Z-\frac{2 N}{g}\right) \\
& \cdot \exp \left\{-S_{F}-S_{C P^{N-1}}+\int_{0}^{\beta} d \tau \int d^{2} r\left(\bar{\eta} p+p^{\dagger} \eta+\bar{Z} J+\bar{J} Z+\frac{g}{2 N} Q_{\mu} A_{\mu}\right)\right\} .
\end{aligned}
$$

Since the action $S_{F}$ is bilinear in the fermionic variables they can easily be integrated out:

$$
S_{F}=-N \operatorname{Tr} \ln \Delta_{F}=-N \operatorname{Tr} \ln \left(\Delta_{F}^{0}+\Delta_{F}^{A}+\Delta_{F}^{K}\right) .
$$

Here we have divided the fermionic contribution into three terms

$$
\begin{aligned}
\Delta_{F}^{0} & =-\frac{1}{2 m} \partial_{r}^{2}+2 g \partial_{\tau} \\
\Delta_{F}^{A} & =-\frac{1}{2 m}\left[i \sigma^{z} 2 \frac{\delta}{\delta Q_{r}} \partial_{r}+i \sigma^{z} \partial_{r}\left(\frac{\delta}{\delta Q_{r}}\right)\right]+2 g i \sigma^{z} \frac{\delta}{\delta Q_{\tau}}+\frac{1}{2 m} \frac{\delta^{2}}{\delta Q_{r}^{2}}, \\
\Delta_{F}^{K} & =i \tilde{\gamma}_{\tau}\left(\frac{g}{2 N}\right) 2\left(B_{\tau} \sigma^{-}+\bar{B}_{\tau} \sigma^{+}\right)+\tilde{\gamma}\left(\frac{g}{2 N}\right)^{2} 4 B_{r} \bar{B}_{r} \\
& +\tilde{\gamma} 2\left(\frac{g}{2 N}\right)\left[2\left(B_{r} \sigma^{-}+\bar{B}_{r} \sigma^{+}\right) \partial_{r}+\partial_{r}\left(B_{r} \sigma^{-}+\bar{B}_{r} \sigma^{+}\right)\right],
\end{aligned}
$$

the first one is the free kinetic part, the second one contains the contributions from the gauge field $A_{\mu}$, which we have substituted by a functional derivative with respect to its source $\left((g / 2 N) A_{\mu} \rightarrow \delta / \delta Q_{\mu}\right)$ as is clear from Eq. (16), and the third term contains the off-diagonal contributions $K_{\mu}$. This division turns out to be useful, since due to the underlying $S U(2)$ structure the $A_{\mu}$ and $K_{\mu}$-terms in $S_{F}$ do not mix within the large- $N$ expansion and we can therefore treat them independently form each other.

We first show that the $K_{\mu}$-terms modify the $C P^{N-1}$ model. An expansion of the non-diagonal part in powers of $1 / \sqrt{N}$ yields: 


$$
\begin{aligned}
S_{F}^{K} & =\int d^{3} p N\left(\frac{g}{2 N}\right)^{2} 4 \bar{B}_{r}(p) B_{s}(-p)\left[\tilde{\gamma} \delta_{r s} \int d^{3} q G_{F}(q)+\frac{1}{2} \tilde{\gamma}^{2} \int d^{3} q G_{F}(q)(2 q+p)_{r}(2 q+p)_{s} G_{F}(q+p)\right] \\
& +\int d^{3} p N\left(\frac{g}{2 N}\right)^{2} 4 \bar{B}_{\tau}(p) B_{\tau}(-p) \frac{\tilde{\gamma}_{\tau}^{2}}{2} \int d^{3} q G_{F}(q) G_{F}(q+p),
\end{aligned}
$$

where $G_{F}(q)=1 /\left(i \nu_{n}-\boldsymbol{q}^{2} / 2 m\right)$ is the fermionic Greens function and where we have introduced the short hand notation $\int d^{3} q=1 / \beta \sum_{\nu_{m}} \int d^{2} q /(2 \pi)^{2}$. Evaluating the integrals in Eq. (21) in the limit $\boldsymbol{p} \rightarrow 0$ and $\omega /|\boldsymbol{p}| \rightarrow 0$ (after proper analytic continuation) and noticing that $(g / 2 N) \bar{B}_{\mu} B_{\nu}=\bar{D}_{\mu}^{B} \bar{Z} D_{\nu}^{B} Z$ one gets for $S_{F}^{K}$ together with the pure bosonic contribution the following generalized $C P^{N-1}$ model:

$$
S_{C P^{N-1}}^{\text {gen. }}=S_{C P^{N-1}}+S_{F}^{K}=\int_{0}^{\beta} d \tau \int d^{2} r\left\{f_{r}^{\delta} \bar{D}_{r}^{B} \bar{Z} D_{r}^{B} Z+\frac{1}{c^{2}} f_{\tau}^{\delta} \bar{D}_{\tau}^{B} \bar{Z} D_{\tau}^{B} Z\right\}
$$

with doping dependent coupling constants

$$
\begin{aligned}
& f_{r}^{\delta}=1+2 g \delta\left(\tilde{\gamma}-2 m \tilde{\gamma}^{2}\right)=1-2 g \delta(1+2 \gamma m) \gamma, \\
& f_{\tau}^{\delta}=1+2 g c^{2} \tilde{\gamma}_{\tau}^{2} \frac{m}{4 \pi} \Theta(\delta),
\end{aligned}
$$

where $\Theta(\delta)=1$ for $\delta>0$ and zero otherwise. Before we can integrate over the bosonic variables in the path integral, we have to decouple the quartic terms. This is achieved by means of the following Hubbard-Stratonovich transformation

$$
\begin{aligned}
& \exp \left\{\frac{g / f_{r}^{\delta}}{2 N} \int_{0}^{\beta} d \tau \int d^{2} r\left[-\left(\bar{Z} \partial_{\mu} Z\right)\left(\bar{Z} \partial_{\mu} Z\right)+i Q_{\mu}\left(\bar{Z} \partial_{\mu} Z\right)\right]\right\} \\
& =\int \mathcal{D} \lambda_{\mu} \exp \left\{\int_{0}^{\beta} d \tau \int d^{2} r\left[-\frac{2}{g / f_{r}^{\delta}} \lambda_{\mu} \lambda_{\mu}+\frac{2 i}{\sqrt{N}} \lambda_{\mu}\left(\bar{Z} \partial_{\mu} Z\right)+\frac{1}{\sqrt{N}} \lambda_{\mu} Q_{\mu}-\frac{g / f_{r}^{\delta}}{8 N} Q_{\mu} Q_{\mu}\right]\right\}
\end{aligned}
$$

where we have rescaled the bosonic fields in such a way that now the constraint $\operatorname{reads} \delta\left(\bar{Z} Z-\frac{2 N}{g / f_{r}^{\delta}}\right)$ and we have absorbed the factor $f_{\tau}^{\delta} / c^{2}$ in a redefinition of the inverse temperature $\left(c / \sqrt{f_{\tau}^{\delta}}\right) \beta \rightarrow \beta$. Since the introduced HubbardStratonovich field $\lambda_{\mu}$ couples now linearly to the source $Q_{\mu}$ it plays from now on the role of the gauge field. Furthermore we include the constraint for the bosonic variables via a lagrange multiplier field $\alpha$ into the action:

$$
\delta\left(|z|^{2}-\frac{2 N}{g / f_{r}^{\delta}}\right)=\int \mathcal{D} \alpha \exp \left\{\int_{0}^{\beta} d \tau \int d^{2} r\left[\frac{i}{\sqrt{N}} \alpha\left(\bar{z} z-\frac{2 N}{g / f_{r}^{\delta}}\right)\right]\right\} .
$$

After these modifications the action is bilinear in the bosonic variables and they can be integrated out. In this way we get the following effective action

$$
S_{\mathrm{eff}}=N \operatorname{Tr} \ln \Delta_{B}-N \operatorname{Tr} \ln \left(\Delta_{F}^{0}+\Delta_{F}^{A}\right)+i \frac{2 \sqrt{N}}{g / f_{r}^{\delta}} \int_{0}^{\beta} d \tau \int d^{2} r \alpha(\boldsymbol{r}, \tau)
$$

with

$$
\begin{aligned}
& \Delta_{B}=-D_{\mu}^{B} D_{\mu}^{B}+M^{2}-\frac{i \alpha}{\sqrt{N}}, \\
& D_{\mu}^{B}=\partial_{\mu}+\frac{i}{\sqrt{N}} \lambda_{\mu} .
\end{aligned}
$$

Please notice that after the steps following Eq. (22) the contributions of the non-diagonal $K_{\mu}$-terms are now contained in the doping dependent coupling constant in front of the last term of Eq. (27). At this stage we have also introduced a mass $M$ for the bosonic variables which is arbitrary since the corresponding mass term $M^{2} \bar{Z} Z$ gives only a constant in the path integral due to the constraint for the bosons [12]. Resubstituting the functional derivative with respect to the source field $Q_{\mu}$ in the fermionic part by the gauge field $\lambda_{\mu}$ and expanding the remaining fermionic part and the bosonic one in powers of $1 / \sqrt{N}$ leads to

$$
S_{\mathrm{eff}}=\sum_{\nu=1}^{\infty} N^{1-\frac{\nu}{2}} S^{(\nu)}
$$


In the limit of large $N$ only the first two terms will survive. The first term is given by

$$
S_{(1)}=i \alpha(\boldsymbol{p}=0, \omega=0)\left(\frac{2}{g / f_{r}^{\delta}}-\int d^{3} q \frac{1}{\boldsymbol{q}^{2}+\nu^{2}+M^{2}}\right),
$$

where $\nu=2 \pi m / \beta$ are bosonic Matsubara frequencies. We want to stress here that without taking into account the non-diagonal terms $\propto K_{\mu}$ there would appear no doping dependent parameter as $f_{r}^{\delta}$ in Eq. (31) since the diagonal fermionic contributions to $S^{(1)}$ are identical to zero due to the vanishing trace of the Pauli matrix $\sigma^{z}$. The second important term in the large $N$-expansion is given by the polarization insertions of the fermions and bosons for the gauge field $\lambda_{\mu}$ as well as for the constraint field $\alpha$ :

$$
S^{(2)}=\frac{1}{2} \int d^{3} p\left\{\lambda_{\mu}\left(\boldsymbol{p}, \omega_{n}\right)\left(\Pi_{\mu \nu}^{B}\left(\boldsymbol{p}, \omega_{n}\right)+\Pi_{\mu \nu}^{F}\left(\boldsymbol{p}, \omega_{n}\right)\right) \lambda_{\nu}\left(-\boldsymbol{p},-\omega_{n}\right)-\alpha\left(\boldsymbol{p}, \omega_{n}\right) F\left(\boldsymbol{p}, \omega_{n}\right) \alpha\left(-\boldsymbol{p},-\omega_{n}\right)\right\},
$$

where the polarization tensors are defined as follows:

$$
\begin{aligned}
\Pi_{\mu \nu}^{B}\left(\boldsymbol{p}, \omega_{n}\right) & =2 \delta_{\mu \nu} \int d^{3} q \frac{1}{\nu_{m}^{2}+\boldsymbol{q}^{2}+M^{2}}-\int d^{3} q \frac{\left(2 q_{\mu}+p_{\mu}\right)\left(2 q_{\nu}+p_{\nu}\right)}{\left[\boldsymbol{q}^{2}+\nu_{m}^{2}+M^{2}\right]\left[(\boldsymbol{q}+\boldsymbol{p})^{2}+\left(\nu_{m}+\omega_{n}\right)^{2}+M^{2}\right]}, \\
\Pi_{\mu \nu}^{F}\left(\boldsymbol{p}, \omega_{n}\right) & =\delta_{\mu r}\left\{2 \delta_{r s} \frac{1}{2 m} \int d^{3} q \frac{1}{i \nu_{m}-\frac{1}{2 m} \boldsymbol{p}^{2}}-\left(\frac{1}{2 m}\right)^{2} \int d^{3} q \frac{(2 p+q)_{r}(2 p+q)_{s}}{\left[i \nu_{m}-\frac{1}{2 m} \boldsymbol{p}^{2}\right]\left[i\left(\nu_{m}+\omega_{n}\right)-\frac{1}{2 m}(\boldsymbol{p}+\boldsymbol{q})^{2}\right]}\right\} \delta_{s \nu} \\
& -\delta_{\mu 0}\left\{\frac{1}{2} \int d^{3} q \frac{1}{\left[i \nu_{m}-\frac{1}{2 m} \boldsymbol{p}^{2}\right]\left[i\left(\nu_{m}+\omega_{n}\right)-\frac{1}{2 m}(\boldsymbol{p}+\boldsymbol{q})^{2}\right]}\right\} \delta_{0 \nu} \\
& -i \frac{1}{2}\left(\frac{1}{2 m}\right) \delta_{\mu 0}\left\{\int d^{3} q \frac{(2 p+q)_{r}}{\left[i \nu_{m}-\frac{1}{2 m} \boldsymbol{p}^{2}\right]\left[i\left(\nu_{m}+\omega_{n}\right)-\frac{1}{2 m}(\boldsymbol{p}+\boldsymbol{q})^{2}\right]}\right\} \delta_{r \nu}, \\
F\left(\boldsymbol{p}, \omega_{n}\right) & =\int d^{3} q \frac{1}{\boldsymbol{q}^{2}+\nu_{m}^{2}+M^{2}} \frac{1}{(\boldsymbol{q}+\boldsymbol{p})^{2}+\left(\nu_{m}+\omega_{n}\right)^{2}+M^{2}} .
\end{aligned}
$$

As a consequence of the local $U(1)$ gauge-symmetry the polarization tensor $\Pi_{\mu \nu}=\Pi_{\mu \nu}^{F}+\Pi_{\mu \nu}^{B}$ has to be transversal $p^{\mu} \Pi_{\mu \nu}=0$ and this property turns out to be very useful in evaluating the polarization tensors by means of a tensorial decomposition.

\section{A. From the renormalized classical to the quantum disordered phase by doping}

Since in the limit $N \rightarrow \infty$ the expression $\exp \left\{-\sqrt{N} S^{(1)}\right\}$ gives a strongly fluctuating factor in the integrand of the generating functional $(16)$ only the contribution $S^{(1)} \equiv 0$ will survive. Evaluating the integral in the expression (31) this saddle point equation establishes a definite relationship between the mass $M$ of the $Z$-bosons, the coupling constant $g$, the temperature $T$, and the doping $\delta$ :

$$
M=\frac{2}{\beta} \operatorname{arcsinh}\left[\frac{1}{2} \exp \left\{-4 \pi \beta\left(\frac{f_{r}^{\delta}}{g}-\frac{1}{g_{c}}\right)\right\}\right]
$$

with $g_{c}=8 \pi / \Lambda$ denoting the critical coupling constant which depends on the ultraviolet cut-off $\Lambda$. Eq. (36) shows that the previously arbitrary mass $M$ is now fixed by the saddle point condition and is therefore dynamically generated [12]. By introducing the renormalized spin stiffness $\rho=2 / g-2 / g_{c}$ at zero doping, where the system is in the Néel ordered phase (at $T=0$ ) [13], we get the following expression for the mass $M$ which is essentially the inverse of the spin-spin correlation length $\xi$

$$
M=\xi^{-1}=\frac{2}{\beta} \operatorname{arcsinh}\left[\frac{1}{2} \exp \{-2 \pi \beta(\rho-4 \gamma(1+2 \gamma m) \delta)\}\right] .
$$

According to Chakravarty, Halperin and Nelson (CHN) [13] we have to distinguish three different regimes depending on the value of the argument $y=\frac{2 \pi}{T}(\rho-4 \gamma(1+2 \gamma m) \delta)$ in the exponential of Eq. (37). For $y \gg 1$ the system is in the renormalized classical (RC) regime where the spin-spin correlation length $\xi$ diverges exponentially as $T$ tends to zero, indicating a ground state with long-range antiferromagnetic Néel order at $T=0(\beta=\infty)$ 


$$
\xi=\beta \exp [2 \pi \beta(\rho-4 \gamma(1+2 \gamma m) \delta)] .
$$

Contrary for $y \ll-1$ the correlation length tends to a constant value at $T=0$

$$
\xi=\frac{1}{4 \pi[4 \gamma(1+2 \gamma m) \delta-\rho]},
$$

implying that the system is in a QD spin-liquid state. From the above discussion it is clear that there must be a phase transition due to doping at $T=0$ where the system goes over from the Néel state to the QD spin liquid state. The corresponding critical doping $\delta_{c}$, where this quantum phase transition occurs, is defined by:

$$
\delta_{c}=\frac{\rho}{4 \gamma(1+2 \gamma m)}
$$

Thus the critical doping $\delta_{c}$ is completely determined by the experimental value of the spin-stiffness $\rho$ at zero doping and the microscopic parameters $m$ and $\gamma$. For an estimate of the critical doping $\delta_{c}$ we use a parameter set [15] of the underlying three-band Hubbard model, which lies in the strong coupling regime, where the spin-fermion model applies. Also this parameter set is very realistic as was shown by quantum Monte Carlo simulations [8] through a comparison of numerical results and experimental data for the cuprates. Furthermore the assumption of isotropic masses and coupling constants in the theory made in the introduction is justified using this parameter set. Using the expressions for the mass $m$ and the coupling constant $\gamma$ given in Ref. [i] we get $m=3 / 2$ and $\gamma=1 / 12$ [15]. With the experimental value $\rho=1.7 \cdot 10^{-2}[16]$ we explicitly get $\delta_{c} \sim 4 \%[11]$.

Finally for $|y| \ll 1$ the system is in the quantum critical (QC) regime which is completely determined by the critical point $\left(\delta_{c}, T=0\right) 114$.

\section{B. Propagator of the gauge field}

After we have discussed the phase diagram of the doped AF we examine here the second term of the large- $N$ expansion, which consists of the polarization insertions of the fermions and bosons for the gauge field $\lambda_{\mu}$ and for the constraint field $\alpha$. As already stated, the polarization tensor (32) of the gauge field $\lambda_{\mu}$ has to be transverse and therefore the following tensorial decomposition is useful [17]:

$$
\Pi_{\mu \nu}(p)=A_{\mu \nu}(p)\left[\Gamma_{1}^{F}(p)+\Gamma_{1}^{B}(p)\right]+B_{\mu \nu}(p)\left[\Gamma_{2}^{F}(p)+\Gamma_{2}^{B}(p)\right],
$$

with $p=\left(\boldsymbol{p}, \omega_{n}\right)$ denoting the wave-vector $\boldsymbol{p}$ together with the Matsubara frequency $\omega_{n}$. Here we have defined the two transverse tensors $\left(p^{2}=\boldsymbol{p}^{2}+\omega_{n}^{2}\right)$

$$
A_{\mu \nu}=\left(\delta_{\mu 0}-\frac{p_{\mu} p_{0}}{p^{2}}\right) \frac{p^{2}}{\boldsymbol{p}^{2}}\left(\delta_{0 \nu}-\frac{p_{0} p_{\nu}}{p^{2}}\right) ; B_{\mu \nu}=\delta_{\mu i}\left(\delta_{i j}-\frac{p_{i} p_{j}}{\boldsymbol{p}^{2}}\right) \delta_{j \nu}
$$

with the following properties:

$$
A_{\mu \nu}+B_{\mu \nu}=\delta_{\mu \nu}-\frac{p_{\mu} p_{\nu}}{p^{2}} ; A_{\mu \nu} B^{\mu \nu}=0
$$

The scalar functions $\Gamma_{1}^{F, B}(p)$ and $\Gamma_{2}^{F, B}(p)$ are evaluated in Appendix $\mathrm{A}$ and are given by:

$$
\begin{aligned}
\Gamma^{B}(p) & :=\Gamma_{1}^{B}(p)=\Gamma_{2}^{B}(p)=\frac{p^{2}+4 M^{2}}{2} F(p)-\frac{M}{4 \pi}, \\
\Gamma_{1}^{F}(p) & =\frac{p^{2}}{\boldsymbol{p}^{2}} \int d^{3} q \frac{1}{\left[\frac{1}{2 m} \boldsymbol{q}^{2}-i \nu_{m}\right]\left[\frac{1}{2 m}(\boldsymbol{q}+\boldsymbol{p})^{2}-i\left(\nu_{m}+\omega_{n}\right)\right]}, \\
\Gamma_{2}^{F}(p) & =\frac{\rho_{F}}{m}-\left(\frac{1}{2 m}\right)^{2} \int d^{3} q \frac{(2 \boldsymbol{q}+\boldsymbol{p}) \cdot(2 \boldsymbol{q}+\boldsymbol{p})}{\left[\frac{1}{2 m} \boldsymbol{q}^{2}-i \nu_{m}\right]\left[\frac{1}{2 m}(\boldsymbol{q}+\boldsymbol{p})^{2}-i\left(\nu_{m}+\omega_{n}\right)\right]} \\
& +\left(\frac{1}{2 m}\right)^{2} \frac{1}{\boldsymbol{p}^{2}} \int d^{3} q \frac{(2 \boldsymbol{q}+\boldsymbol{p}) \cdot \boldsymbol{p}(2 \boldsymbol{q}+\boldsymbol{p}) \cdot \boldsymbol{p}}{\left[\frac{1}{2 m} \boldsymbol{q}^{2}-i \nu_{m}\right]\left[\frac{1}{2 m}(\boldsymbol{q}+\boldsymbol{p})^{2}-i\left(\nu_{m}+\omega_{n}\right)\right]} .
\end{aligned}
$$


After an analytical continuation to real frequencies $i \omega_{n} \rightarrow \omega+i 0^{+}$and evaluating the various integrals at $T=0$ we get the following expressions for the scalar $\Gamma$ functions (The details of this calculation are delegated to Appendix B):

$$
\begin{aligned}
& \Gamma^{B}(p)=\frac{\boldsymbol{p}^{2}-\omega^{2}+4 M^{2}}{8 \pi \sqrt{\boldsymbol{p}^{2}-\omega^{2}}} \arcsin \left(\sqrt{\frac{\boldsymbol{p}^{2}-\omega^{2}}{4 M^{2}+\left(\boldsymbol{p}^{2}-\omega^{2}\right)}}\right)-\frac{M}{4 \pi}, \\
& \Gamma_{1}^{F}(p)=\frac{p^{2}}{\boldsymbol{p}^{2}} \frac{m}{2 \pi}\left(1-\frac{x \Theta\left(x^{2}-1\right)}{\sqrt{x^{2}-1}}+i \frac{x \Theta\left(1-x^{2}\right)}{\sqrt{1-x^{2}}}\right), \\
& \Gamma_{2}^{F}(p)=\frac{\rho_{F}}{m}\left[x^{2}+\left(x-x^{3}\right) \frac{\Theta\left(x^{2}-1\right)}{\sqrt{x^{2}-1}}-i\left(x-x^{3}\right) \frac{\Theta\left(1-x^{2}\right)}{\sqrt{1-x^{2}}}\right],
\end{aligned}
$$

where $x=\left|\frac{\omega}{\boldsymbol{p}_{v_{F}}}\right| . \rho_{F}$ is the fermionic density and $v_{F}$ the Fermi velocity. Within the Coulomb gauge, $\boldsymbol{\nabla} \cdot \boldsymbol{\lambda}=0$, we get for the propagator of the gauge field $\lambda_{\mu}$

$$
\begin{aligned}
D_{00}^{\lambda} & =\frac{p^{2}}{\boldsymbol{p}^{2}}\left(\Gamma^{B}(\boldsymbol{p}, \omega)+\Gamma_{1}^{F}(\boldsymbol{p}, \omega)\right)^{-1}, \\
D_{0 i}^{\lambda} & =0 \\
D_{i j}^{\lambda} & =\left(\delta_{i j}-\frac{p_{i} p_{j}}{\boldsymbol{p}^{2}}\right)\left(\Gamma^{B}(\boldsymbol{p}, \omega)+\Gamma_{2}^{F}(\boldsymbol{p}, \omega)\right)^{-1} .
\end{aligned}
$$

Since we are interested on the long-wavelength low-frequency physics of the gauge theory it is appropriate to study the limit $\boldsymbol{p} \rightarrow 0, \omega \rightarrow 0$. Because of the step functions in the fermionic contributions we have to distinguish two different regions in the $\omega-p$ plane.

In the region, where $x>1$, we get

$$
\begin{aligned}
& D_{\tau \tau}^{\lambda^{>}}=\frac{\omega^{2}}{\boldsymbol{p}^{2}} \frac{-M}{\frac{1}{24 \pi} \omega^{2}+\frac{\rho_{F} M}{m}}, \\
& D_{i j}^{\lambda^{>}}=\left(\delta_{i j}-\frac{p_{i} p_{j}}{\boldsymbol{p}^{2}}\right) \frac{-M}{\frac{1}{24 \pi} \omega^{2}-\frac{\rho_{F} M}{2 m}}
\end{aligned}
$$

and for $x<1$ we get:

$$
\begin{aligned}
D_{\tau \tau}^{\lambda^{<}} & =\frac{1}{\frac{1}{24 \pi M} \boldsymbol{p}^{2}+\frac{m}{2 \pi}}, \\
D_{i j}^{\lambda^{<}}(\boldsymbol{p}, \omega) & =\left(\delta_{i j}-\frac{p_{i} p_{j}}{\boldsymbol{p}^{2}}\right) \frac{M}{\frac{1}{24 \pi} \boldsymbol{p}^{2}-i \rho_{F} \frac{M}{m}\left|\frac{\omega}{\boldsymbol{p}_{v_{F}}}\right|} .
\end{aligned}
$$

The propagator of the constraint field $\alpha$ is completely determined by the bosonic polarization and is therefore independent of the value of $x$

$$
D^{\alpha}=F_{B}^{-1}=8 \pi M
$$

As we see from Eqs. (53) - (57) the time component of the gauge field propagator as well as the propagator of the Lagrange-multiplier field $\alpha$ are massive, since they are Debeye screened, and thus mediate only short-range densitydensity interactions between the fermions and bosons. Here we want to remark that the pole in the prefactor $\frac{\omega^{2}}{\boldsymbol{p}^{2}}$ in Eq. (53) is not physical, since it is a consequence of the choosen Coulomb gauge. In fact one can explicitly see in the axial or in the Lorentz gauge, that the singularity due to that prefactor in Eq. (53) is absent. Physical poles of the gauge field propagator are only given by the zeros of the gauge independent combinations of the fermionic and bosonic scalar functions $\left(\Gamma^{B}(\boldsymbol{p}, \omega)+\Gamma_{1}^{F}(\boldsymbol{p}, \omega)\right)$ and $\left(\Gamma^{B}(\boldsymbol{p}, \omega)+\Gamma_{2}^{F}(\boldsymbol{p}, \omega)\right)$ appearing in the corresponding expressions for the propagator.

In the region $\left|\frac{\omega}{\boldsymbol{p}_{F}}\right|>1$ the spatial component of the gauge field propagator has a pole at a finite frequency $\omega^{2}=24 \pi \frac{\rho_{F} M}{m}$ with a form reminiscent of the plasmon pole in an electron gas. Furthermore, in the opposite limit, $\left|\frac{\omega}{\boldsymbol{p} v_{F}}\right|<1$, the gauge field propagator turns out to be massless and describes an overdamped mode with a dispersion law $\omega \propto i p^{3}$, whose physical origin can be traced back to the Landau damping $\propto i \omega / p$ due to fermions near the Fermi surface. As a consequence of this massless mode the fermions and bosons experience an effective long-range interaction 
by exchanging transverse gauge bosons. The form of the propagator in Eq. (56) is essentially the same as found by Nagaosa and Lee [18] and Reizer [19], with a central difference given by the fact that the mass $M$ of the magnetic excitations determines the strength of the propagator. Hence as a special feature of the present theory a new energy scale, the spin-gap $\Delta \propto M$ measured in neutron scattering experiments [20], enters in a natural way and appears as the strength of the interaction. Therefore, we expect that the long-range current-current interaction mediated by the transverse gauge field becomes only effective in the QD phase, where the spin-gap $\Delta$ is large compared to the thermal energy $k_{B} T$. This implies that the tendency to form bound states only occurs in the disordered magnetic state, such that the phenomena of charge-spin separation and pairing in this theory should be restricted to that region.

\section{DISCUSSION AND SUMMARY}

As was noticed first by Holstein et al. 21] and later by Reizer [19], the coupling of fermions to a transverse vector gauge field leads to deviations from the Fermi-liquid picture. However, in the case of a conventional metal interacting with the electromagnetic field, the effects of the exchange of transverse gauge bosons are in practice unobservable since they are suppressed by the ratio of the Fermi-velocity and the speed of light $\left(\frac{v_{F}}{c}\right)^{2}$. In contrast to those systems, in the above gauge theory for the doped AF the coupling constant is of order unity and therefore no such small parameter enters, a feature common to gauge theories developed recently for strongly correlated metals 18,22,23]. Calculating the self-energy for the fermions at $\boldsymbol{k}_{F}$ in lowest order of perturbation theory one gets an unusual frequency dependence

$\Sigma \propto \omega^{\frac{2}{3}}$, which implies that the quasi-particle weight $Z=\left|1-\frac{\partial}{\partial \omega} \operatorname{Re} \Sigma_{\omega \rightarrow 0}\right|^{-1}$ vanishes at the Fermi surface signaling a break down of Fermi-liquid picture [18], and hence, a perturbative expansion in the coupling constant is in this case not valid [23] and one has to implement some non-perturbative approaches. In spite of the conflicting claims in the recent literature concerning the one-particle properties of gauge theories for strongly correlated metals [24], we would like to point out that the results obtained there are not directly applicable to our case, since the physically relevant one-particle properties appear only after a rotation back to the global refence frame. This amounts in the lowest order of the large $N$-expansion to a convolution of the free fermionic and bosonic propagators and the corrections due to the gauge fields are obtained at $\mathcal{O}(1 / N)$. Results pertinent to this point will be presented elsewhere [25]. An even stronger difference with the general discussion of gauge theories in this context should be expected due to the possibility of formation of bound states, that we discuss in the following.

The singular mode of the gauge field mediates a long-range current-current interaction between the $Z$-bosons and the fermions, which in the static limit reduces to a logarithmic potential. Moreover since the strength of the singular gauge field propagator is proportional to the spin-gap $\Delta$ we expect the formation of bound states only in the QD phase, where the spin-gap is large compared to the thermal energy $k_{B} T$. Hence particles with opposite charge will bind and the low-energy sector of the physical spectrum of the theory will contain only states with zero charge with respect to the gauge field $\lambda$. Since the charge of the fields is given by their spin-projection, the bound states correspond to spin-singlets. According to table (13) the following three bound states are possible:

(a) Bound states between two bosons:

$$
\bar{Z}-Z
$$

These bound states correspond to spin-waves excitations (remember $\boldsymbol{n}=\bar{Z} \boldsymbol{\sigma} Z$ ) at the antiferromagnetic wave vector $\boldsymbol{Q}=(\pi, \pi)$ with a gap $\Delta \propto M$ in the spectrum.

(b) Bound states between a fermion and a boson:

$$
Z-p
$$

These bound states have an electric charge $+e$ but have no spin. Thus they describe spinless charge-excitation with fermionic character. Thus, this scenario gives an alternative way to charge-spin separation, where the bare excitations are just spin- $\frac{1}{2}$ fermions but the renormalized ones are spinless.

(c) Bound states between two fermions:

$$
p_{\downarrow}-p_{\uparrow}
$$

Such a bound state describes the pairing of two fermions in a spin-singlet with charge $2 e$. Hence, charge-spin separation and pairing are intimately connected in our case and result from the same interaction. 
A similar description for holes in a spin-liquid state was derived by Wen [26] starting with a one-band Hubbard model. However, in that case charge and spin degrees of freedom are separated from the very beginning (the bare fermions do not carry spin) and hence the phenomenon of charge-spin separation does not follow from the formation of bound states as in our theory. Quite on the contrary, the bound states between fermions and bosons in that theory carry charge $e$ and spin, implying that charge-spin separation only occurs when there is no long-range interaction present, i. e. when either the gauge field acquires a mass due to a Chern-Simons term or abnormal screening at a high doping level takes place [27].

For the bound states (58)-(50) we get the following picture. At a doping level which is greater than the critical one, there is a crossover from the QC phase at high temperatures to the QD phase at low $T$. Below the crossover temperature $T^{*}$ the bound states will form and this should be reflected by various physical quantities.

The fact that the formation of the bound states brings the system towards a singlet state, may explain the anomalous data in nuclear magnetic relaxation experiments [28], where a strong reduction of both the Knight-shift and the relaxation-rate on oxygen and copper is observed as a function of temperature, well above the superconducting transition, for underdoped samples of YBCO. Closely connected with this is the observation of a spin-gap in neutron scattering experiments on YBCO [20] which corresponds to the mass of the $Z$-bosons. Moreover, when the bound states between a fermion and a boson form, charge-spin separation takes place and the scattering of the chargecarriers by the gauge fields vanishes since the new bound state has zero charge and therefore does not couple to the gauge field. Such a scenario is consistent with recent resistivity measurements in underdoped YBCO 123 [29,30] and 124 [31], where a reduction of the resistivity with respect to the linear temperature dependence is observed, at a temperature $T^{*}$ where the nuclear relaxation rate $\left(1 / T_{1} T\right)$ on $\mathrm{Cu}$ shows a maximum that is commonly associated with the spin-gap [28]. Furthermore in the resistivity perpendicular to the $\mathrm{CuO}$-planes a crossover from a metallic-like to a semiconductor-like behavior is observed at the same characteristic temperature $T^{*}$ [30], which in our theory follows from the fact that one has to break the bound states in order to have charge transport perpendicular to the planes. This is also consistent with a suppression of the optical conductivity parallel to the $c$-axis that was reported recently for underdoped samples of 123 [32] indicating the opening of a pseudo gap along the $c$-axis.

In conclusion we have shown that within the long-wavelength, low-energy sector of the spin-fermion model a quantitative correct description for the doping induced quantum phase transition appearing in the HTSC materials can be obtained. Furthermore, in the gauge field theory of the doped AF in the rotating reference frame, charge-spin separation and pairing follow in a natural way from the formation of bound state in the QD phase (above the critical doping and at low temperatures) due to the long-range force mediated by the singular mode of the transverse gauge field, such that an intimate connection between these phenomena and the spin-gap results. Finally, we have also discussed the connection of these bound states with the anomalous features observed in the low-doping regime of the cuprates.

We acknowledge support by the Deutsche Forschungsgemeinschaft under Project No. Mu 820/5-2.

\section{APPENDIX A: TENSORIAL DECOMPOSITION}

(a) Evaluation of $\Gamma_{1}^{F}$

Due to the properties (43) of the two transversal tensors $A_{\mu \nu}$ and $B_{\mu \nu}$ we get $\left(p^{2}=\boldsymbol{p}^{2}+\omega_{n}^{2}\right)$

$$
\left(\delta_{\mu 0}-\frac{p_{\mu} p_{0}}{p^{2}}\right) \frac{p^{2}}{\boldsymbol{p}^{2}}\left(\delta_{0 \nu}-\frac{p_{0} p_{\nu}}{p^{2}}\right) \Pi_{\mu \nu}^{F}(p)=\frac{p^{2}}{\boldsymbol{p}^{2}} \Pi_{00}^{F}(p)=\Gamma_{1}^{F}(p),
$$

where $\Pi_{00}(p)$ follows from the expression (34) of the fermionic contribution to the polarization tensor:

$$
\Pi_{00}(p)=\frac{1}{\beta} \sum_{\nu_{m}} \int \frac{d^{2} q}{(2 \pi)^{2}} \frac{1}{\left[\frac{1}{2 m} \boldsymbol{q}^{2}-i \nu_{m}\right]\left[\frac{1}{2 m}(\boldsymbol{q}+\boldsymbol{p})^{2}-i\left(\nu_{m}+\omega_{n}\right)\right]}=: I_{2}^{F}(p) .
$$

(b) Evaluation of $\Gamma_{2}^{F}$

From (43) further follows:

$$
\delta_{\mu i}\left(\delta_{i j}-\frac{p_{i} p_{j}}{\boldsymbol{p}^{2}}\right) \delta_{j \nu} \Pi_{\mu \nu}^{F}=\left(\delta_{i j}-\frac{p_{i} p_{j}}{\boldsymbol{p}^{2}}\right) \Pi_{i j}^{F}=\Pi_{i i}^{F}-\frac{p_{i} p_{j}}{\boldsymbol{p}^{2}} \Pi_{i j}^{F}=\Gamma_{2}^{F},
$$


with

$$
\begin{aligned}
\Pi_{i i}^{F} & =\frac{\delta_{i i}}{m} \frac{1}{\beta} \sum_{\nu_{m}} \int \frac{d^{2} q}{(2 \pi)^{2}} \frac{1}{\frac{1}{2 m} \boldsymbol{q}^{2}-i \nu_{m}}-\left(\frac{1}{2 m}\right)^{2} \frac{1}{\beta} \sum_{\nu_{m}} \int \frac{d^{2} q}{(2 \pi)^{2}} \frac{(2 \boldsymbol{q}+\boldsymbol{p}) \cdot(2 \boldsymbol{q}+\boldsymbol{p})}{\left[\frac{1}{2 m} \boldsymbol{q}^{2}-i \nu_{m}\right]\left[\frac{1}{2 m}(\boldsymbol{q}+\boldsymbol{p})^{2}-i\left(\nu_{m}+\omega_{n}\right)\right]} \\
& =: \frac{\delta_{i i}}{m} I_{1}^{F}(p)-\left(\frac{1}{2 m}\right)^{2} I_{3}^{F}(p)
\end{aligned}
$$

and

$$
\begin{aligned}
p_{i} p_{j} \Pi_{i j}^{F} & =\frac{1}{m} \boldsymbol{p}^{2} \frac{1}{\beta} \sum_{\nu_{m}} \int \frac{d^{2} q}{(2 \pi)^{2}} \frac{1}{\frac{1}{2 m} \boldsymbol{q}^{2}-i \nu_{m}} \\
& -\left(\frac{1}{2 m}\right)^{2} \frac{1}{\beta} \sum_{\nu_{m}} \int \frac{d^{2} q}{(2 \pi)^{2}} \frac{(2 \boldsymbol{q}+\boldsymbol{p}) \cdot \boldsymbol{p} \quad(2 \boldsymbol{q}+\boldsymbol{p}) \cdot \boldsymbol{p}}{\left[\frac{1}{2 m} \boldsymbol{q}^{2}-i \nu_{m}\right]\left[\frac{1}{2 m}(\boldsymbol{q}+\boldsymbol{p})^{2}-i\left(\nu_{m}+\omega_{n}\right)\right]} \\
& =: \frac{1}{m} \boldsymbol{p}^{2} I_{1}^{F}(p)-\left(\frac{1}{2 m}\right)^{2} I_{4}^{F}(p)
\end{aligned}
$$

\section{APPENDIX B: FERMIONIC AND BOSONIC INTEGRALS}

In this appendix we calculate the bosonic and fermionic integrals appearing in the scalar functions $\Gamma^{B}, \Gamma_{1}^{F}$ and $\Gamma_{2}^{F}$ of the polarization tensor for the gauge field.

First we evaluate the bosonic integral $F\left(\boldsymbol{p}, \omega_{n}\right)$ defined by:

$$
F\left(\boldsymbol{p}, \omega_{n}\right)=\frac{1}{\beta} \sum_{\tilde{\nu}} \int \frac{d^{2} q}{(2 \pi)^{2}} \frac{1}{\left[\boldsymbol{q}^{2}+\nu_{m}^{2}+M^{2}\right]\left[(\boldsymbol{q}+\boldsymbol{p})^{2}+\left(\nu_{m}+\omega_{n}\right)^{2}+M^{2}\right]} .
$$

Using a Feynman parametrisation we obtain:

$$
F\left(\boldsymbol{p}, \omega_{n}\right)=\frac{1}{\beta} \sum_{\tilde{\nu}} \int \frac{d^{2} q}{(2 \pi)^{2}} \int_{0}^{1} d x \frac{1}{\left[M^{2}+\boldsymbol{q}^{2}+\tilde{\nu}^{2}+\boldsymbol{p}^{2} x(1-x)+\omega^{2} x(1-x)\right]^{2}}
$$

where $\tilde{\nu}=\nu+\omega(1-x)$. Integration over momentum leads to:

$$
F\left(\boldsymbol{p}, \omega_{n}\right)=\frac{1}{\beta} \sum_{\tilde{\nu}} \frac{1}{4 \pi} \int_{0}^{1} d x \frac{1}{M^{2}+\left(\boldsymbol{p}^{2}+\omega^{2}\right) x(1-x)+\tilde{\nu}^{2}} .
$$

In order to evaluate the frequency sum we make a partial fraction decomposition of the integrand. (Here we have to introduce a factor $\exp (i \nu \eta)$ to ensure convergence of the series):

$$
\begin{aligned}
& F\left(\boldsymbol{p}, \omega_{n}\right)= \lim _{\eta \rightarrow 0} \int_{0}^{1} \frac{d x}{4 \pi} \frac{1}{\beta} \sum_{\tilde{\nu}} \frac{1}{2 \sqrt{M^{2}+\left(\boldsymbol{p}^{2}+\omega_{n}^{2}\right) x(1-x)}} \exp (i \nu \eta) \cdot \\
&\left\{\frac{1}{i \nu+\left(\sqrt{M^{2}+\left(\boldsymbol{p}^{2}+\omega_{n}^{2}\right) x(1-x)}+i \omega_{n}(1-x)\right)}-\frac{1}{i \nu-\left(\sqrt{M^{2}+\left(\boldsymbol{p}^{2}+\omega_{n}^{2}\right) x(1-x)}-i \omega_{n}(1-x)\right)}\right\} \\
&= \int_{0}^{1} \frac{d x}{4 \pi} \frac{1}{2 \sqrt{M^{2}+\left(\boldsymbol{p}^{2}+\omega_{n}^{2}\right) x(1-x)}} \\
&\left\{1+\frac{1}{\exp \left[\beta \sqrt{M^{2}+\left(\boldsymbol{p}^{2}+\omega_{n}^{2}\right) x(1-x)}-i \beta \omega_{n}(1-x)\right]-1}+\frac{1}{\exp \left[\beta \sqrt{M^{2}+\left(\boldsymbol{p}^{2}+\omega_{n}^{2}\right) x(1-x)}+i \beta \omega_{n}(1-x)\right]-1}\right\}
\end{aligned}
$$

For $T \rightarrow 0$ the temperature dependent part of the integral gives only an exponentially small correction and can therefore be neglected. After an analytical continuation to reel frequencies $i \omega_{n} \rightarrow \omega+i \eta$ we get: 


$$
F(\boldsymbol{p}, \omega) \stackrel{T \rightarrow^{0}}{\simeq} \frac{1}{4 \pi} \frac{1}{\sqrt{\boldsymbol{p}^{2}-\omega^{2}}} \arcsin \left(\sqrt{\frac{\boldsymbol{p}^{2}-\omega^{2}}{4 M^{2}+\left(\boldsymbol{p}^{2}-\omega^{2}\right)}}\right) .
$$

In the following we calculate the fermionic integrals. The first one is gives and equals the density of fermions at the fermi surface:

$$
I_{1}^{F}=\frac{1}{\beta} \sum_{\nu_{m}} \int \frac{d^{2} q}{(2 \pi)^{2}} \frac{1}{\frac{1}{2 m} \boldsymbol{q}^{2}-i \nu_{m}}=-\rho_{F} .
$$

For the calculation of the remaining fermionic integrals we make use of the formal identity

$$
\frac{1}{\omega \pm i \eta}=\mathcal{P} \frac{1}{\omega} \mp i \pi \delta(\omega)
$$

with $\mathcal{P}$ denoting the principal value. In this way we obtain

$$
\begin{aligned}
I_{2}^{F} & =\frac{1}{\beta} \sum_{\nu_{m}} \int \frac{d^{2} q}{(2 \pi)^{2}} \frac{1}{\left[\frac{1}{2 m} \boldsymbol{q}^{2}-i \nu_{m}\right]\left[\frac{1}{2 m}(\boldsymbol{q}+\boldsymbol{p})^{2}-i \nu_{m}-(\omega+i \eta)\right]} \\
& =-\mathcal{P} \int \frac{d^{2} q}{(2 \pi)^{2}} \frac{n(\boldsymbol{q}-\boldsymbol{p} / 2)-n(\boldsymbol{q}+\boldsymbol{p} / 2)}{\omega-(\boldsymbol{q} \cdot \boldsymbol{p}) / m}+i \pi \int \frac{d^{2} q}{(2 \pi)^{2}}[n(\boldsymbol{q}-\boldsymbol{p} / 2)-n(\boldsymbol{q}+\boldsymbol{p} / 2)] \delta(\omega-(\boldsymbol{q} \cdot \boldsymbol{p}) / m) .
\end{aligned}
$$

Furthermore, we go over to zero temperature, where the difference between the two Fermi distributions is given by

$$
n(\boldsymbol{q}-\boldsymbol{p} / 2)-n(\boldsymbol{q}+\boldsymbol{p} / 2)=-\boldsymbol{p} \cdot \boldsymbol{\nabla} n(\boldsymbol{q}) \stackrel{T=0}{=} \boldsymbol{p} \cdot \boldsymbol{q} / q \delta\left(|\boldsymbol{q}|-k_{F}\right) .
$$

Using this expression we get

$$
\begin{aligned}
I_{2}^{F} & =-\mathcal{P}\left(\frac{1}{2 \pi}\right)^{2} 2 \int_{0}^{\pi} d \theta \int_{0}^{\infty} d q \delta\left(q-k_{F}\right) \frac{q p \cos \theta}{\omega-(q p \cos \theta) / m} \\
& +i \frac{1}{2 \pi} \int_{0}^{\pi} d \theta \int_{0}^{\infty} d q q p \cos \theta \delta\left(q-k_{F}\right) \delta(\omega-(q p \cos \theta) / m) \\
& =2 m\left(\frac{1}{2 \pi}\right)^{2} \mathcal{P} \int_{-1}^{1} d z \frac{z}{z-x} \frac{1}{\sqrt{1-z^{2}}}+i\left(\frac{1}{2 \pi}\right) m \int_{-1}^{1} d z \delta(z-x) \frac{z}{\sqrt{1-z^{2}}} \\
& =\frac{m}{2 \pi}\left(1-\frac{x \Theta\left(x^{2}-1\right)}{\sqrt{x^{2}-1}}+i \frac{x \Theta\left(1-x^{2}\right)}{\sqrt{1-x^{2}}}\right) ; x=\left|\frac{\omega}{\boldsymbol{p} v_{F}}\right| .
\end{aligned}
$$

The remaining fermionic integrals are calculated in the same way and we just give the results here

$$
\begin{aligned}
I_{3}^{F} & =\frac{1}{\beta} \sum_{\nu_{m}} \int \frac{d^{2} q}{(2 \pi)^{2}} \frac{(2 \boldsymbol{q}+\boldsymbol{p}) \cdot(2 \boldsymbol{q}+\boldsymbol{p})}{\left[\frac{1}{2 m} \boldsymbol{q}^{2}-i \nu_{m}\right]\left[\frac{1}{2 m}(\boldsymbol{q}+\boldsymbol{p})^{2}-i \nu_{m}-(\omega+i \eta)\right]} \\
& =4 k_{F}^{2} I_{2}^{F}
\end{aligned}
$$

and

$$
\begin{aligned}
I_{4}^{F} & =\frac{1}{\beta} \sum_{\nu_{m}} \int \frac{d^{2} q}{(2 \pi)^{2}} \frac{(2 \boldsymbol{q}+\boldsymbol{p}) \cdot \boldsymbol{p}(2 \boldsymbol{q}+\boldsymbol{p}) \cdot \boldsymbol{p}}{\left[\frac{1}{2 m} \boldsymbol{q}^{2}-i \nu_{m}\right]\left[\frac{1}{2 m}(\boldsymbol{q}+\boldsymbol{p})^{2}-i \nu_{m}-(\omega+i \eta)\right]} \\
& =\frac{m}{2 \pi} 4 k_{F}^{2} \boldsymbol{p}^{2}\left(x^{2}+\frac{1}{2}-\frac{x^{3} \Theta\left(x^{2}-1\right)}{\sqrt{x^{2}-1}}+i \frac{x^{3} \Theta\left(1-x^{2}\right)}{\sqrt{1-x^{2}}}\right) .
\end{aligned}
$$

[1] Y. Endoh et al., Phys. Rev. B 37 (1988) 7443. 
[2] N.P. Ong, Physica C 235-240 (1994) 221; B. Batlogg et al., ibid. p. 130; For general reviews: Physical Properties of High Temperature Superconductors, Vols. 1-3, ed. D.M. Ginsberg (World Scientific, Singapore, 1991).

[3] G. Shirane et al., Physica B 197 (1994) 158.

[4] C. Kübert and A. Muramatsu, Phys. Rev. B 47 (1993) 787.

[5] P. Prelovšek, Phys. Lett. A 126 (1988) 287; J. Zaanen and A.M. Olés, Phys. Rev. B 37 (1988) 4923; A. Muramatsu, R. Zeyher, and D. Schmeltzer, Europhys. Lett. 7 (1988) 473.

[6] A. Angelucci and G. Jug, Int. Jour. Mod. Phys. B 3 (1989) 1069.

[7] B.I. Shraiman and E.D. Siggia, Phys. Rev. Lett. 61 (1988) 467; Phys. Rev. B 41 (1990) 350; ibid. 42 (1990) 2485.

[8] G. Dopf, A. Muramatsu and W. Hanke, Phys. Rev. Lett. 68 (1992) 353; Europhys. Lett. 17 (1992) 559; G. Dopf et al., Phys. Rev. Lett. 68 (1992) 2082; A. Muramatsu et al., Festkörperprobleme Volume 32 (Vieweg 1992) p. 317.

[9] R. Rajamaran, Solitons and instantons (North-Holland, Amsterdam, 1987).

[10] P.J. Hilton, An Introduction to Homotopy Theory (Cambridge University Press, Cambridge, 1961); A.M. Polyakov, Gauge Fields and Strings (Harwood Academic Publishers, Chur, 1987).

[11] C. Kübert and A. Muramatsu, to be published in Europhys. Lett.

[12] A. D'Adda, M. Lüscher, and P. Di Vecchia, Nucl. Phys. B 146 (1978) 63.

[13] S. Chakravarty, B.I. Halperin, and D.R. Nelson, Phys. Rev. B 39 (1989) 2344.

[14] S. Sachdev and J. Ye, Phys. Rev. Lett. 69 (1992) 2411; A.V. Chubukov and S. Sachdev, Phys. Rev. Lett. 71 (1993) 169; A.V. Chubukov, S. Sachdev, and J. Ye, Phys. Rev. B 49 (1994) 11919.

[15] The underlying parameter of the three-band Hubbard model are: $U=6, \Delta=4$ and $t_{p p}=-0.5$. Within this parameter set $J_{k}=3 / 4, \bar{t}=-0.5$ and $\tilde{t}=-1 / 8$. Note that all parameters are given in units of the hopping between the $\mathrm{Cu}$ and $\mathrm{O}$ orbitals $t_{p d}=1.3 \mathrm{eV}$ of the original 3-band Hubbard model.

[16] M. Greven et al., Phys. Rev. Lett. 72 (1994) 1096; here $\rho=1.7 \cdot 10^{-2}$ is given in units of $t_{p d}$, which corresponds to the experimental value $2 \pi \rho=140 \mathrm{meV}$.

[17] N. Dorey and N.E. Mavromatos, Nucl. Phys. B 386 (1992) 614.

[18] N. Nagaosa and P.A. Lee, Phys. Rev. Lett. 64 (1990) 2450.

[19] M. Reizer, Phys. Rev. B 39 (1989) 1602; 40 (1989) 11571.

[20] V. Barzykin, D. Pines, A. Sokol, and D. Thelen, Phys. Rev. B 49 (1994) 1548; J. Rossat-Mignod et al., Physica C 185-189 (1991) 86; L.P. Regnault et al., Physica C 235-240 (1994) 59.

[21] T. Holstein, R.E. Norton, and P. Pincus, Phys. Rev. B 8 (1973) 2649.

[22] J. Polchinski, Nucl. Phys. B 422 [FS] (1994) 617.

[23] B. Blok and H. Monien, Phys. Rev. B 47 (1993) 3454.

[24] D.V. Khveshchenko and P.C. Stamp, Phys. Rev. Lett. 71 (1993) 2118; H.J. Kwon, A. Houghton, and J.B. Marston, SISSA preprint cond-mat/9501067; B.L. Altshuler, L.B. Ioffe, and A.J. Millis, Phys. Rev. B 50 (1994) 14048.

[25] F. Hamelbeck, C. Kübert and A. Muramatsu, unpublished.

[26] X.G. Wen, Phys. Rev. B 39 (1989) 7223.

[27] X.G. Wen and A. Zee, Nucl. Phys. B 326 (1989) 619.

[28] M. Takigawa et al., Phys. Rev. B 43 (1991) 247.

[29] B. Bucher et al., Phys. Rev. Lett. 70 (1993) 2012.

[30] K. Takenaka et al., Phys. Rev. B 50 (1994) 6534.

[31] T. Ito, K. Takenaka, and S. Uchida, Phys. Rev. Lett. 70 (1993) 3995.

[32] C.C. Homes et al., Phys. Rev. Lett. 71 (1993) 1645. 